[0212-7199 (2005) 22: 4; pp 167-171] ANALES DE MEDICINA INTERNA Copyright (C) 2005 ARAN EDICIONES, S.L.

An. MED. INTERNA (Madrid) Vol. 22, N. ${ }^{\circ} 4$, pp. 167-171, 2005

\section{Prevención de eventos cardiovasculares en hipertensos mayores de 65 años bajo el cuidado de un programa de control. Estudio de cohorte}

\author{
J. DÍAZ, F. ACHILLI, S. FIGAR, G. WAISMAN, E. LANGLOIS, C. GALARZA, \\ L. CAMERA, F. GONZÁLEZ B. DE QUIRÓS
}

Programa de Medicina Geriátrica. Sección de Hipertensión Arterial. Servicio de Clínica Médica y Gerencia Médica. Hospital Italiano de Buenos Aires. Buenos Aires. Argentina

PREVENTION OF CARDIOVASCULAR DISEASES BY AN ANTIHYPERTENSIVE PROGRAM IN THE ELDERLY. COHORT STUDY

\section{RESUMEN}

Introducción: Iniciamos en el 2000 un programa de control para la hipertensión arterial.

Objetivo: Conocer el riesgo de morir y de presentar eventos cardiovasculares en los hipertensos bajo el cuidado del programa.

Método: Se conformó una cohorte de 1.922 mayores de 65 años en agosto del 2000. Se consideró hipertenso si se conocía como tal, si recibía fármacos antihipertensivos o si tenía dos tomas de presión $\geq 140 / 90$ $\mathrm{mmHg}$. Evento cardiovascular a las internaciones por enfermedad coronaria, insuficiencia cardiaca y accidente cerebrovascular. Expresamos densidad de incidencia (DI) de mortalidad y de eventos cardiovasculares cada 100 persona-años en hipertensos y en no hipertensos y practicamos regresión de cox para ajustar por factores de riesgo en estudio. Se compara el tiempo al primer evento cardiovascular y a la muerte con Log Rank Test.

Resultados: Edad 77,6 ( \pm 5,4) años, 73,9\% mujeres, el 48,3\% eran hipertensos, diferentes significativamente con respecto a los normotensos en edad $79( \pm 5)$ vs. $77( \pm 5) \mathrm{p}<0,001$, porcentaje de diabéticos $(16,1$ vs. 7,6\%) $\mathrm{p}<0,001$. La DI del total de eventos fue de 1,86 vs. 3,02 (RR 1,62, IC95\% 1,09-2,42) La DI de mortalidad fue de 2,2 vs. 2,1 (RR 1.04, IC95\% 0,69-1,58) Tiempo promedio de seguimiento 28 meses, el porcentaje de pacientes libre de eventos a este tiempo fue de $93 \%$ (hta) vs. $96 \%$ (no hta) (p < 0,001). En el multivariado fueron significativas: diabetes, dislipidemia, tabaquismo, edad y sexo, perdiendo peso la hipertensión arterial, OR 1.30 (IC95\% 0,86-1,98).

Conclusiones: La hipertensión no incrementó el riesgo de presentar eventos cardiovasculares en nuestros hipertensos a 2,3 años.

PALABRAS CLAVE: Hipertensión arterial. Ancianos. Estudio de cohorte.

\begin{abstract}
Introduction: We started on year 2000 a Complex Intervention Program addressed at hypertension control among our patients.

Aim: To compare the risk of cardiovascular events and of dying in hypertensive patients under Program care.

Method: We started follow-up of a cohort of 1922 patients over 65 years in August 2000. Hipertension diagnosis was ascertained if patient reported to be hypertensive, or was under anti hypertensive treatment or if he/she had two blood pressure measurements $\geq 140 / 90 \mathrm{~mm} \mathrm{Hg}$. Cardiovascular events were considered to be admissions due to coronary disease, cardiac insufficiency or stroke. Incidence is reported by 100 person years follow-up. Relative risks between hypertensive and normotensive patients were calculated and Cox regresión was used to adjust for potential confounders. We compared time to first cardiovascular event and to death with Log Rank Test.

Results: Fourty eight point three percent of patients were hypertensive and differed from normotensive patients as to age (79 (5) years vs. 77 (5) $p$ $<0.001$, proportion of diabetic patients $(16.1 \%$ vs. $7.6 \% p<0.001)$ Mean follow-up time was 28 months. Mortality RR was 1.04 (95\% CI 0.691.58). As to incidence of cardiovascular events it was 1.86 in normotensive vs. 3.02 (RR 1.62 95\% CI 1.09-2.42). When adjusted by age, sex, smoking, dislipemia and diabetes, OR was 1.3 (95\% CI 0.86-1.98)

Conclusions: Hypertension did not increase the risk in cardiovascular events among our hypertensive patients at 2.3 years follow-up.
\end{abstract}

KEY WORDS: Hipertensión arterial. Elderly. Cohort study.

Díaz J, Achilli F, Figar S, Waisman G, Langlois E, Galarza C, González B de Quirós F. Prevención de eventos cardiovasculares en hipertensos mayores de 65 años bajo el cuidado de un programa de control. Estudio de cohorte. An Med Interna (Madrid) 2005; 22: 167-171.

\section{INTRODUCCIÓN}

La hipertensión arterial y sus complicaciones, el accidente cerebrovascular, la insuficiencia cardiaca, el infarto de miocardio y la insuficiencia renal crónica, permanecen como uno de los problemas más comunes y devastadores de la salud, que experimentan las personas de mayor edad.
La hipertensión arterial es la enfermedad más prevalente en la población, su frecuencia aumenta con la edad siendo del $26-36 \%$ en la población general y llegando al 60-70\% en los mayores de 65 años (1-5). El 65-75\% de los casos de hipertensión arterial en pacientes de edad avanzada corresponde a la variedad sistólica aislada $(6,7)$.

Un estudio realizado en la ciudad autónoma de Buenos 
Aires sobre una muestra randomizada del padrón de la Obra Social de los Jubilados de la Argentina, en la población de mayores de 65 años, demostró que la prevalencia de hipertensión arterial era del 77,5\%, el 54\% de ellos recibían tratamiento farmacológico y sólo el $18,5 \%$ tenía controlada su hipertensión arterial (8).

Desde la óptica epidemiológica el mayor número de eventos ocurre entre los pacientes con hipertensión leve por ser en este rango en el que se distribuye la mayor proporción de enfermos.

Actualmente el tratamiento debe ser más agresivo y pretender valores de presión arterial más bajos cuanto más daño de órgano blanco exista. Reducciones de $5 \mathrm{mmHg}$ para la presión diastólica y de $10 \mathrm{mmHg}$ para la sistólica reducen el riesgo de eventos cerebrovasculares en un $36 \%$ y en un $15 \%$ los eventos coronarios $(6,9,10)$.

Pero a pesar de la evidencia existe aun una gran brecha con la práctica clínica (11) entre lo demostrado en ensayos controlados y los resultados de estudios transversales que evalúan el grado de control en las distintas poblaciones de hipertensos. Sin duda los resultados no satisfactorios incluyen un componente de incumplimiento del paciente, relacionado con la edad y el nivel de educación; un segundo componente de inercia clínica y otro de las características de las enfermedades crónicas.

Para sortear estas barreras, se decidió implementar en nuestro hospital un programa para el manejo de la hipertensión utilizando las herramientas del gerenciamiento médico. El programa se desarrolló en varias etapas y requirió distintas evaluaciones.

En la primera se realizó un corte transversal durante agosto a diciembre del año 2000 de 8.000 pacientes para conocer la prevalencia e iniciar el tamizaje de la población. Se determinó que el $68 \%$ de nuestra población ambulatoria mayor a 65 años eran hipertensos con un grado de control del $55 \%$ (12).

Posteriormente un estudio clínico controlado en 500 hipertensos evaluó la eficacia a los 12 meses de este programa evidenciando una mejora del $20 \%$ en el grado de control y una reducción de $11 \mathrm{mmHg}$ en la presión sistólica en el grupo de hipertensos no controlados $(13,14)$.

Sin embargo los programas de manejo de enfermedades crónicas requieren mantener el impacto de sus intervenciones a lo largo del tiempo, es por eso que en esta etapa evaluativa analizamos el riesgo de morir y de presentar eventos cardiovasculares a 2.3 años en los pacientes hipertensos bajo el cuidado de nuestro programa.

\section{MÉTODO}

\section{POBLACIÓN}

Se seleccionaron los primeros 1.000 pacientes hipertensos y los primeros 1.000 no hipertensos detectados en el estudio transversal de prevalencia de hipertensión arterial. Este estudio se realizó en pacientes ambulatorios mayores de 65 años, de ambos sexos, elegidos aleatoreamente de la sala de espera de nuestra institución. Se consideraron hipertensos a aquellos pacientes que se conocían como tales, a los que estaban en tratamiento farmacológico para la hipertensión o a los que tenían dos tomas separada por al menos 7 días de presión arterial mayores a 140/90 $\mathrm{mmHg}$.
El 96\% aceptó entrar en el estudio de seguimiento, conformándose una cohorte, estratificada por el riesgo de ser hipertenso o no, de 1.922 pacientes seguida desde agosto de 2000 hasta diciembre de 2002.

\section{DISEÑO}

Estudio de cohorte prospectivo.

\section{INTERVENCIÓN}

Los médicos son intervenidos por el programa a través de discusión de guías de manejo en reuniones periódicas. Un recordatorio en la historia clínica electrónica le informa sobre el estado de la presión arterial, la adherencia a la dieta hiposódica y al ejercicio físico. Los pacientes hipertensos son marcados habitualmente por el sistema informático que les avisa a las recepcionistas que el paciente debe pasar por el consultorio del programa antes de ver al médico.

Un monitor, alumno avanzado de medicina evalúa a los hipertensos periódicamente y a los controles en el consultorio del programa. La toma de la presión arterial se realiza con tensiómetros OMROM-431, en tres tomas consecutivas separadas de un minuto y con un descanso previo de cinco minutos. A los hipertensos conocidos y nuevos se les entrega un folleto explicativo de los cuidados y los riesgos de la hipertensión y se los invita a un taller de cuatro clases. Estas clases son dictadas por especialistas en hipertensión y son orientadas a cambiar hábitos y comportamiento, descubrir la sal invisible, conocer los alimentos protectores y combatir de manera sencilla el sedentarismo (15).

\section{MEDIDAS DE RESULTADOS}

Resultado primario: presentar algún evento cardiovascular durante el período comprendido entre agosto de 2000 y diciembre de 2002. Se consideraron como eventos cardiovasculares las internaciones, por: enfermedad coronaria (infarto y angina inestable), insuficiencia cardiaca (ICC) y accidente cerebrovascular (ACV). Los datos se obtuvieron de bases de datos pertenecientes al departamento de información hospitalaria; para la identificación de estos se valió del sistema de codificación por ICD9 (International Clasification of Diseases, Ninth versión)

Resultado secundario: mortalidad por cualquier causa durante el período de seguimiento.

Se registraron los casos incidentes de hipertensión arterial en los no hipertensos basales, definidos con dos tomas separadas al menos por 7 días mayores a 139/89 mmHg y los de diabetes en ambos subgrupos según la presencia del registro médico en las evoluciones o por presentar una hemoglobina glicosilada mayor a $5,6 \mathrm{mg} / \mathrm{dl}$.

\section{ANÁLISIS ESTADÍSTICO}

Para el análisis univariado se calcularon las densidades de incidencia de mortalidad por cualquier causa, de eventos cardiovasculares totales, de ICC, enfermedad coronaria y ACV. 
Las tasas se expresan cada 100 persona-años en cada subgrupo, se compararon los riesgos con la razón de las tasas (Rate Ratio) y esta se expresa con su IC $95 \%$.

Se realizó un análisis de sobrevida con Kaplan Meier al primer evento cardiovascular y otro a la muerte comparando ambos grupos (hipertensos vs. no hipertensos) con Log Rank Test.

El análisis multivariado para el resultado primario se realizó con regresión de Cox ajustando ser hipertenso con las variables edad, sexo, dislipidemia, diabetes y tabaquismo actual.

Se expresa el promedio de todas las tomas de presión arterial realizadas durante cada semestre y se comparan con el test de Anova, las diferencias entre los cinco semestres se determinan en el análisis post hoc con el test de Bonferroni.

Las variables continuas de las características basales se expresan con media y desvío standard, y se comparan con $\mathrm{t}$ test, las proporciones se expresan en porcentaje e IC 95\% y se comparan con Chi cuadrado.

\section{RESULTADOS}

Edad 77,6 (5,4) años, 73,9\% mujeres, 928 eran hipertensos, de los cuales $44 \%$ no estaban controlados (20\% hiperten- sos sistodiastólicos y $80 \%$ sistólicos), siendo diferentes significativamente con respecto a los normotensos en edad $79( \pm 5)$ vs. $77( \pm 5)$, en el porcentaje de diabéticos y en el de dislipidemia. No se encontraron diferencias entre sexo, tabaquismo y ACV previo (Tabla I).

Tiempo promedio de seguimiento de la cohorte: 27 meses. El 88\% de los pacientes completó el seguimiento.

La densidad de incidencia para eventos cardiovasculares totales fue de 1,86 en los no hipertensos vs. (versus) 3,02 en los hipertensos; la de ACV fue 0,6 vs. 1,02 respectivamente, para enfermedad coronaria fue de 1,77 en el grupo de no hipertensos vs. 2,44 en el grupo de hipertensos mientras que para ICC fue de 0,23 vs. 0,92 respectivamente. La densidad de incidencia de mortalidad en cada grupo fue de 2,19 vs. 2,10. La razón entre las tasas para cada resultado se expresa en la tabla II.

El tiempo promedio en meses libre de eventos cardiovasculares fue similar para ambos grupos, no hipertensos 28,67 (IC 95\% 28,43-28,92) vs. hipertensos 28,33 (IC 95\% 28,04$28,63)$. A los 2,5 años de seguimiento el porcentaje de pacientes que no sufrieron eventos fue de $96 \%$ vs. 93\% respectivamente (Log rank test 6,61 p < 0,001) (Fig. 1). El porcentaje de pacientes vivos fue de $94 \%$ en los no hipertensos vs. $95 \%$ en los hipertensos ( Log rank test 0,01 p = 0,91).

En el análisis multivariado para eventos cardiovasculares

TABLA I

CARACTERÍSTICAS BASALES

\begin{tabular}{lccc}
\hline Variables & $\begin{array}{c}\text { Hipertensos } \\
n: 928\end{array}$ & $\begin{array}{c}\text { Normotensos } \\
n: 994\end{array}$ & $p$ \\
\hline Edad en años, media (SD) & $78,6(5,3)$ & $76,6(5,2)$ & $<0,001$ \\
Sexo $\mathrm{F}, \mathrm{n} \%$ & $679(73,1 \%)$ & $742(74,6 \%)$ & 0,42 \\
Diabetes, $\mathrm{n} \%$ & $120(12,9 \%)$ & $47(4,7 \%)$ & $<0,001$ \\
Tabaquismo, n\% & $135(14,5 \%)$ & $142(14,3 \%)$ & 0,89 \\
ACV previo, n \% & $11(1,18 \%)$ & $6(0,6 \%)$ & 0,17 \\
Dislipemia, n \% & $581(62,6 \%)$ & $467(46,98 \%)$ & $<0,001$ \\
$\begin{array}{l}\text { Presión arterial sistólica } \\
\quad \text { en mmHg, media (SD) }\end{array}$ & $139,2(20,05)$ & $122,5(15,5)$ & $<0,001$ \\
Presión arterial diastólica & & & $<0,001$ \\
$\quad$ en mmHg, media(SD) & $75,09(10,8)$ & $69,8(9,4)$ & \\
\hline
\end{tabular}

\begin{tabular}{|c|c|c|}
\hline \multicolumn{3}{|c|}{ TABLA II } \\
\hline \multicolumn{3}{|c|}{$\begin{array}{l}\text { RAZÓN DE LAS TASAS DE LOS RESULTADOS CON SUS } \\
\text { IC } 95 \%\end{array}$} \\
\hline Resultado & $\begin{array}{l}\text { ón de tasas } \\
\text { te Ratio) }\end{array}$ & IC $95 \%$ \\
\hline \multicolumn{3}{|l|}{ Eventos } \\
\hline cardiovasculares totales & 1,62 & $1,09-2,42$ \\
\hline \multicolumn{3}{|l|}{ Accidentes } \\
\hline cerebrovasculares & 1,57 & $0,8-3,09$ \\
\hline Enfermedad coronaria & 1,38 & $0,9-2,1$ \\
\hline Insuficiencia cardiaca & 3,98 & $1,49-10,66$ \\
\hline Mortalidad por cualquier causa & 1,04 & $0,69-1,58$ \\
\hline
\end{tabular}

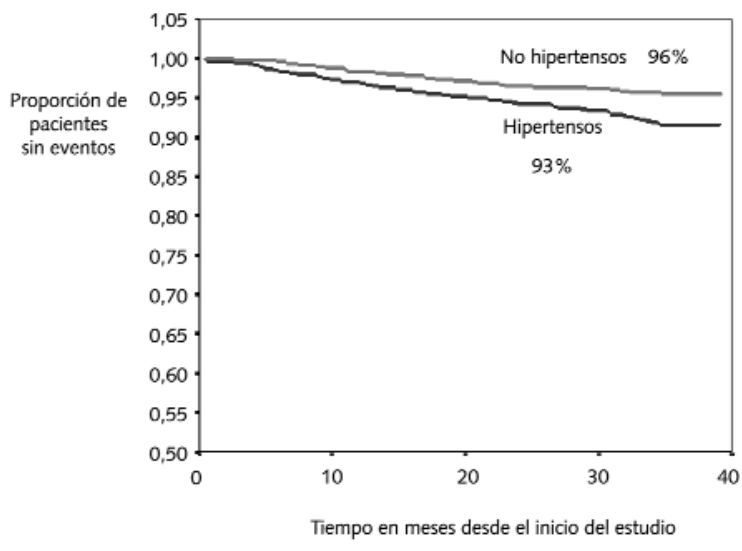

Fig. 1. Gráfico de sobrevida: proporción de pacientes libres de eventos en el seguimiento. 
totales presentó relevancia sexo masculino, edad, diabetes, dislipidemia y tabaquismo, perdiendo significancia estadística ser hipertenso (Tabla III). No se encontraron interacción entre las distintas variables.

La incidencia anual de diabetes fue de 1,35 en los no hipertensos vs. 1,41 en los hipertensos, (RR 0,95; IC95\% $0,57-1,60)$. Se desarrollaron $166(16 \%)$ casos nuevos de hipertensos entre los inicialmente normotensos durante el periodo de observación.

La presión arterial sistólica descendió $3 \mathrm{mmHg}$ en los hipertensos, $(\mathrm{p}<0,001)$, en el análisis post hoc se observó que la diferencia se encontró entre el último periodo y el primero (136 mmHg vs. $139 \mathrm{mmHg}$, p 0,03); y el último y el segundo (136 mmHg vs. $139 \mathrm{mmHg}, \mathrm{p} \mathrm{0,03).}$

\section{TABLA III}

ANÁLISIS MULTIVARIADO PARA EVENTOS CARDIOVASCULARES

\begin{tabular}{lcccc}
\hline & & & \multicolumn{2}{c}{ IC 95\% } \\
\cline { 4 - 5 } & $\mathrm{p}$ & OR & Min. & Máx. \\
\hline HTA & 0,204 & 1,309 & 0,864 & 1,982 \\
Diabetes & 0,009 & 1,904 & 1,172 & 3,093 \\
Tbq & 0,050 & 1,625 & 1,000 & 2,639 \\
Dislipemia & 0,008 & 1,796 & 1,165 & 2,771 \\
Edad & 0,000 & 1,069 & 1,031 & 1,109 \\
Sexo M & 0,011 & 1,738 & 1,134 & 2,665 \\
\hline
\end{tabular}

En el grupo normotenso la presión arterial sistólica subió 5 mmHg. $(\mathrm{p}<0,001)$ siendo diferente el periodo $1 \mathrm{vs}$. el 3, 4, 5 (122 mm Hg vs. $128 \mathrm{~mm} \mathrm{Hg}$, vs. $128 \mathrm{~mm} \mathrm{Hg}$ vs. $127 \mathrm{~mm} \mathrm{Hg}$, $\mathrm{p}<0,001$ para cada par de comparaciones) y el 2 vs. 3, 4, 5 (122 mm Hg vs. $128 \mathrm{~mm} \mathrm{Hg}$, vs. $128 \mathrm{mmHg}$, vs. $127 \mathrm{~mm} \mathrm{Hg}$, p $<0,001$ para cada par de comparaciones) (Fig. 2).

En cuanto al grado de control alcanzado a dos años del inicio de la cohorte, este llega al 69\% (número de tomas: 628).

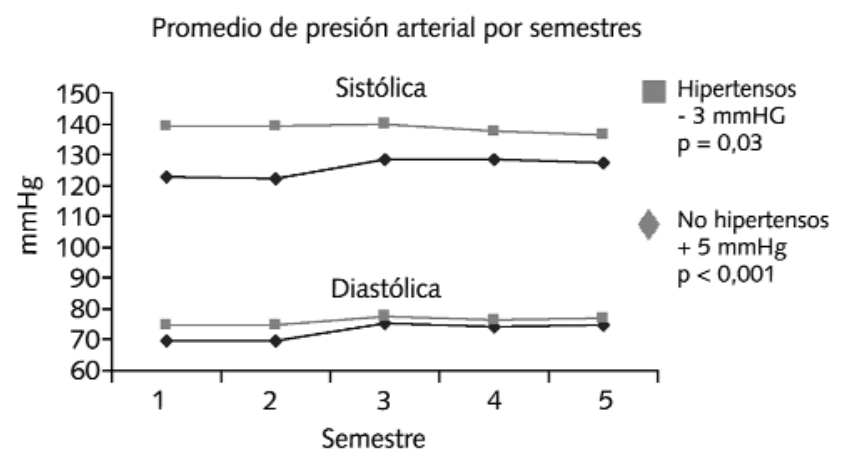

Fig. 2. Evolución de la presión arterial en el tiempo.

\section{DISCUSIÓN}

El principal resultado de nuestro trabajo es que si bien los hipertensos tienen mas riesgo de eventos cardiovasculares que los normotensos a 2,3 años, no se debió la hipertensión.

La diferencia de riesgo atribuida a la hipertensión para eventos cardiovasculares encontrada en el análisis univariado no se evidencia cuando ajustamos con otras variables.

Este es un resultado alentador dado que es sabido que de todos los factores de riesgo cardiovasculares la hipertensión es el de mayor peso (16).

Este estudio no se diseñó para conocer los predictores de eventos cardiovasculares y muerte en nuestra población añosa, sin embargo puso de manifiesto la necesidad de intensificar nuestras intervenciones dirigidas al manejo de la diabetes, tabaquismo y dislipidemia.

Al igual que en la literatura, la edad y el sexo masculino fueron también variables con significancia estadística para el desarrollo de eventos cardiovasculares.

Coincidentemente con lo publicado la hipertensión generó mas riesgo para accidentes cerebrovasculares que para eventos coronarios, la enfermedad coronaria resultó ser el principal contribuyente a los eventos totales en ambos grupos, creemos que no se observaron diferencias en las incidencias por falta de número muestral. Sin embargo alcanzamos poder estadístico para evidenciar el peso de la hipertensión como generador de mayor riesgo de insuficiencia cardiaca.

También nuestros datos son coincidentes en mostrar que el control de la hipertensión en mayores de 65 años disminuye la morbilidad mas que la mortalidad (17). Esto se ve además reflejado en el porcentaje de pacientes libres de eventos $\mathrm{y}$ en el porcentaje de pacientes que sobreviven a los dos años.

La incidencia anual de diabetes, nuevos casos en los pacientes que al momento de la visita basal no eran diabéticos, fue similar al estudio LIFE (18).

Con respecto a los nuevos hipertensos en este grupo etáreo, la incidencia fue de 7 casos nuevos cada 100 pacientes, abundan en la literatura estudios de prevalencia de hipertensión arterial y no encontramos en nuestro país ningún estudio de incidencia.

Pequeños descensos de presión arterial reducen sustancialmente la carga de enfermedad cardiovascular, en estudios poblacionales se observa que una reducción de $2 \mathrm{mmHg}$ disminuye en un $6 \%$ el riesgo de muerte por ACV y en un $4 \%$ el de por coronoariopatia $(19,20)$, esto podría explicar la disminución del riesgo obtenida por el descenso de presión en nuestros hipertensos.

Sin embargo debemos tener en cuenta que el ascenso de $5 \mathrm{mmHg}$ de la presión sistólica en los no hipertensos basales, nos alerta de incentivar medidas higiénico dietéticas en estos pacientes que son de alto riesgo para devenir hipertensos.

Los pequeños cambios observados en la diastólica no fueron analizados por producirse en rangos normales y dado que en los pacientes añosos el riesgo se ve aumentado a expensas de la sistólica.

El hecho de que a 2,3 años la hipertensión no le agregue mas riesgo de eventos a nuestros hipertensos con respecto a los normotensos puede deberse a varios factores. Sin embargo ocurren bajo el funcionamiento del programa por lo que creemos que este estudio observacional apoya la continuidad del mismo dado que se basa en medidas de bajo costo donde el cambio más importante sobre el cual se sustenta es el trabajo en equipo. 


\section{Bibliografía}

1. Burt VL, et al. Trends in the prevalence, awareness, treatment, and control of hypertension in the adult US population. Data from the health examination surveys, 1960 to 1991. Hypertension 1995; 26 (1): 60-9.

2. Echeverría RF, et al. Prevalence of arterial hypertension in La Plata, Argentina. Buenos Aires: Medicina 1988; 48 (1): 22-8.

3. Echeverria RF, et al. Knowledge and treatment of hypertension in La Plata, Argentina. Buenos Aires: Medicina 1989; 49 (1): 53-8.

4. De Lena SM, et al. Prevalence of arterial hypertension in a rural population of Buenos Aires. Buenos Aires: Medicina 1995; 55 (3): 225-30.

5. Burt VL, et al. Prevalence of hypertension in the US adult population. Results from the Third National Health and Nutrition Examination Survey, 1988-1991. Hypertension 1995; 25 (3): 305-13.

6. Franklin SS, et al. Hemodynamic patterns of age-related changes in blood pressure. The Framingham Heart Study. Circulation 1997; 96 (1): 308-15.

7. National High Blood Pressure Education Program Working Group Report on Hypertension in the Elderly. National High Blood Pressure Education Program Working Group. Hypertension 1994; 23 (3): 27585.

8. Przygoda P, et al. Lack of effective blood pressure control among an elder hypertensive population in Buenos Aires. Am J Hypertens 1998; 11 (8 Pt 1): 1024-7.

9. Collins R, et al. Blood pressure, stroke, and coronary heart disease. Part 2, Short-term reductions in blood pressure: overview of randomised drug trials in their epidemiological context. Lancet 1990; 335 (8693): 827-38.

10. Prevention of stroke by antihypertensive drug treatment in older persons with isolated systolic hypertension. Final results of the Systolic Hypertension in the Elderly Program (SHEP). SHEP Cooperative Research Group. Jama 1991; 265 (24): 3255-64.

11. Phillips L, Branch W, Cook C, Doyle J, El Kebbi I, Gallina D. Inercia Clínica. Ann Intern Med 2001; 135: 825-834.

12. Janson J, Figar S, González B, De Quiros F, Waisman G, Galarza C, et al. Prevalence of hypertension among ambulatory elderly patients in a private university hospital in argentina. J Hypertens 2002; 20 (Supl. 4): S85.

13. CAPE. Complex Antihypertensive Program in the Elderly, 2001. http://www.hospitalitaliano.org.ar/cape

14. Figar S, Waisman G, González B, de Quiros F, Galarza C, Marchetti M, et al. Narrowing the gap in hypertension: Effectiveness of a Complex Antihypertensive Program in the Elderly. Disease Management 2004; 7 : 235-244.

15. Aprender Salud. http://www.hospitalitaliano.org.ar/comunidad.

16. Pocok SJ, McCormac KV, Gueyffier F, et al. A score for predicting risk of death from cardiovascular disease in adults with raised blood pressure. BMJ 2001; 323: 75-81.

17. Kaplan N. Hypertension trials: 1990 to 2000. Curr Opin Nephrol Hypertens. 2001; 10 (4): 501-5.

18. Dahlof B, Devereux R, Kjeldsen S, et al. LIFE. The Lancet 2002; 359: 23.

19. Whelton P, He J, Appel L. Primary Prevention of Hypertension. Jama 2002; 288: 16

20. National High Blood Pressure Education Program Working Group report on primary prevention of hypertension. Arch Intern Med 1993; 153: $186-208$. 